04

\title{
Моделирование коронального источника жесткого рентгеновского излучения в турбулентной плазме солнечных вспышек
}

\author{
(C) Ю.Е. Чариков, А.Н. Шабалин
}

Физико-технический институт им. А.Ф. Иофрфе РАН, 194021 Санкт-Петербург, Россия

e-mail: taoastronomer@gmail.com

Поступило в Редакцию 1 февраля 2021 г.

В окончательной редакции 15 марта 2021 г.

Принято к публикации 17 марта 2021 г.

Рассмотрена кинетика электронных пучков, ускоренных в столкновительной плазме солнечных (звездных) вспышек с учетом стационарной ионно-звуковой моды, локализованной в вершине магнитной петли, и магнитных флуктуаций. Астрофизический аспект процесса распространения связан с интерпретацией жесткого рентгеновского излучения в плазме вспышечных петель. Показано, что при плотности плазмы в корональной части вспышечных петель Солнца, не превышающей $10^{10} \mathrm{~cm}^{-3}$, учет дополнительного рассеяния на ионно-звуковой моде с отношением плотности энергии турбулентности к тепловой энергии плазмы $\sim 5 \cdot 10^{-5}-10^{-3}$ и магнитных флуктуациях с уровнем $5 \cdot 10^{-2}$ не приводит к появлению яркого источника жесткого рентгеновского излучения в корональной части петли в модели изотропного распределения ускоренных электронов. В анизотропном случае при жестком спектре электронов корональный источник, при наличии ионно-звуковой турбулентности, может существовать непродолжительное время после начала генерации турбулентности. И только в случае мягкого спектра ускоренных электронов (показатель степенного спектра > 5) и относительно высокой концентрации плазмы в вершине магнитной петли $>10^{10} \mathrm{~cm}^{-3}$ происходит генерация яркого коронального источника на энергиях $25-50 \mathrm{keV}$ вне зависимости от питч-углового распределения ускоренных электронов в источнике. Показано значительное влияние турбулентности на распределение линейной степени поляризации жесткого рентгеновского излучения вдоль петли, приводящее к уменьшению экстремальных значений в корональной части на 5-35\%. Интегральные значения поляризации не превышают $10 \%$.

Ключевые слова: турбулентность, кинетика электронных пучков, жесткое рентгеновское излучение, магнитная петля.

DOI: 10.21883/JTF.2021.08.51092.20-21

\section{Введение}

Традиционно источники жесткого рентгеновского (ЖР) излучения преимущественно наблюдались в хромосфере, в основаниях магнитных петель [1]. В последние годы на космических аппаратах Yohkoh и RHESSI зарегистрированы источники ЖР излучения с максимумами яркости не только в основаниях, но также над вершиной петли [2] и/или в вершине петли [3-5]. Из-за низкой концентрации плазмы в вершине они менее яркие и потому наблюдаются намного реже при относительно малом динамическом диапазоне рентгеновских и гаммаспектрометров. Заметим, что отношение концентраций плазмы в основаниях и вершине петли может составить 3-4 порядка. Поток ЖР излучения [6] пропорционален концентрации плазмы и количеству ускоренных электронов в локальной области источника. Следовательно, для обеспечения высокой рентгеновской яркости ЖР излучения при реальных значениях концентрации плазмы в вершине магнитных петель высокоэнергичные электроны должны накапливаться в вершине магнитной петли. Причины накопления связаны с механизмами рассеяния и питч-угловым распределением ускоренных электронов $[7,8]$. В настоящей работе рассматривает- ся влияние стационарной волновой моды на эффект накопления ускоренных электронов в верхней части магнитной петли во время солнечных вспышек. Рассматривается ионно-звуковая мода, которая возбуждается в плазме магнитной петли возникающим обратным электрическим током при условии неизотермичности температуры электронов и ионов, что выполняется, по крайней мере, на масштабе релаксационного времени. Условием возникновения турбулентности в результате присутствия обратного тока в плазме является превышение скорости дрейфа электронов фоновой плазмы над скоростью ионного звука, когда становится возможным черенковское возбуждение ионно-звуковой турбулентности. Такое превышение зависит от параметров пучка электронов и плазмы петли, т.е. от конкретной модели (см. таблицу) и реализуется с небольшим запаздыванием по отношению к началу инжекции ускоренных электронов (см. ниже). Рассматривается случай слабой турбулентности, плотность энергии которой составляет $10^{-5}-10^{-3}$ от плотности тепловой энергии $n k_{\mathrm{B}} T_{e}$ в корональной части петли с генерацией в области сильного индукционного электрического поля, в которой возможно превышение пороговых значений скорости дрейфа 
электронов обратного тока. Здесь $n$ и $T_{e}-$ концентрация и температура плазмы, $k_{\mathrm{B}}-$ постоянная Больцмана. В такой постановке задачи будет оценено влияние стационарной по величине ионно-звуковой турбулентности на распределение ускоренных электронов и источников ЖР излучения с учетом возможной локализации пульсаций, вызванной наличием максимумов в распределении индукционного электрического поля $[9,10]$. Таким образом, в настоящей работе не рассматривается обратное влияние функции распределения электронов на уровень турбулентности ионно-звуковых волн и возможная зависимость от времени плотности энергии турбулентности. Нестационарными являются времена начала, окончания и области генерации, которые связаны с экстремумами в распределении величины индукционного электрического поля вдоль петли.

\section{1. Коэффициент диффузии ионно-звуковой турбулентности}

В кинетическом уравнении (формула 1 в работе [11]) коэффициент, ответственный за угловое рассеяние ускоренных электронов на ионно-звуковых волнах, имеет вид [12] $D^{s}=D^{t r} / p_{e}^{2}=\left(v / v_{i s}\right)^{2} D_{l} / p_{e}^{2}$, где $D^{t r}, D_{l}-$ поперечная и продольная составляющие коэффициента турбулентной диффузии для ионного звука в изотропной плазме, $p_{e}$ - импульс электрона, $v-$ скорость электрона, $v_{i s}$ - фазовая скорость ионно-звуковых волн, $v_{i s}^{2}=k_{\mathrm{B}} T_{e} / m_{p}$, где $m_{p}-$ масса протона. Для температуры в корональной части петли $10^{6}-10^{7} \mathrm{~K}$ скорость ионного звука $\sim 10^{7}-3 \cdot 10^{7} \mathrm{~cm} / \mathrm{s}$, т. е. отношение $v / v_{i s} \approx 10^{3}$ для электрона с энергией $30 \mathrm{keV}$. Coгласно $[13,14]$, продольный коэффициент ионно-звуковой турбулентности определяется, как

$$
\begin{aligned}
D_{l}= & \frac{15 \sqrt{\pi}}{8 \sqrt{2}} Z^{2} m_{e}^{2} v_{T_{e}}^{5}\left(\frac{m_{e}}{m_{i}}\right)^{\frac{3}{2}} \frac{T_{e}}{T_{i}} k_{g} v_{T_{e}}\left(\frac{1}{2 E c^{2}}\right)^{\frac{3}{2}} \\
& \times\left[\sqrt{\frac{Q}{Q_{*}^{s}}}+e^{-\sqrt{\frac{Q}{Q_{*}^{s}}}}-1\right],
\end{aligned}
$$

где мощность источника плазмонов определяется полной плотностью энергии ионно-звуковых колебаний, $T_{i}$ - температура ионной компоненты плазмы. В настоящей работе предполагается стационарный по величине уровень ионно-звуковой турбулентности с отношением плотности энергии $W^{s} / n k_{\mathrm{B}} T_{e}$ в диапазоне значений $5 \cdot 10^{-5}-10^{-3}$, что влечет постоянство отношения $Q / Q_{*}^{s}$, где $Q_{*}^{s}$ - мощность генерации ионного звука в режиме насыщения $Q_{*}^{s}=(15 / 16) k_{g} v_{s} n T_{e}\left(m_{e} / m_{i}\right)\left(T_{e} / T_{i}\right)$. Для соотношения температур $T_{i} / T_{e}=0.1$, $W^{s} / n k_{\mathrm{B}} T_{e}=5 \cdot 10^{-5}$, значение $Q / Q_{*}^{s}=2 \cdot 10^{-4}$. Волновое число $k_{g}$ определяет область волновых чисел, для которых генерация плазмонов превосходит их бесстолкновительное поглощение Ландау, т.е. удовлетворяет условию $k_{g} d_{e} \approx 0.1$, где $d_{e}-$ радиус Дебая. Радиус
Дебая в плазме с $T_{e}=10^{7} \mathrm{~K}, T_{i}=10^{6} \mathrm{~K}$ и концентрацией плазмы $10^{10} \mathrm{~cm}^{-3}$ равен $d_{e}=0.07 \mathrm{~cm}$. В этом случае затухание ионно-звуковых волн мало ввиду малого количества резонансных ионов.

\section{2. Вспышка SOL2013-05-15T01:45}

Рассмотрим результаты ультрафиолетовых (УФ) и ЖР наблюдений с высоким пространственным разрешением. В качестве примера источника ЖР-излучения в вершине петли на рис. 1 показаны изображения вспышки SOL2013-05-15T01:45 класса X1 в УФ излучении в линии $193 \AA$ с наложенными контурами мягкого и жесткого рентгеновского излучения (рис. 1,a). Структура магнитного поля напоминает высокую петлю с каспом и систему более низких магнитных петель в виде аркады. Отчетливо виден источник ЖР излучения в корональной части магнитной структуры. Динамическое отношение потоков из различных боксов показано на рис. $1, c$. Боксы для построения данных отношений отмечены на изображении в диапазоне 25-50 keV (рис. 1, b). Локальные источники отмечены цифрами $0,1,2$.

УФ излучение во время вспышки локализуется в тонких трубках, связанных с магнитным потоком. Поскольку разрешение в рентгеновском диапазоне ниже, чем в УФ диапазоне, локализация рентгеновских источников обнаруживает некоторые боксы, которые локализуются в определенных частях УФ структуры. Бокс 2 ассоциирован нами с областью каспа аркады, расположенного высоко в короне. Яркость коронального ЖР источника на энергиях $12-25$ и 25-50 keV в сравнении с яркостью южного (бокс 1) и, отдельно, северного (бокс 0) оснований аркады оценена на рис. 1, $c$ и составляет $>20 \%$ в зависимости от времени и диапазона энергии.

\section{3. Моделирование коронального источника ЖР излучения}

Наиболее важный вывод, следующий из подобного анализа для ряда вспышек, зарегистрированных RHESSI, - это наличие источника ЖР излучения в верхних (корональных) частях магнитных петель. Интерпретация ЖР источника в вершине предполагает накопление как ускоренных, так и тепловых электронов в этой части петель. В данном рассмотрении эффектом возможного испарения плазмы в верхнюю часть петли пренебрежем для того, чтобы более отчетливо выявить роль ионно- звуковой моды в локализации ускоренных электронов. Инжекция ускоренных электронов в расчетах производится в вершине петли, в ее корональной части. Поскольку задача кинетики пучка электронов и вспышечной плазмы является многопараметрической, приведем в таблице наборы значений для рассмотренных моделей.

В задаче распространения ускоренных электронов в плазме возникает индукционное электрическое по- 


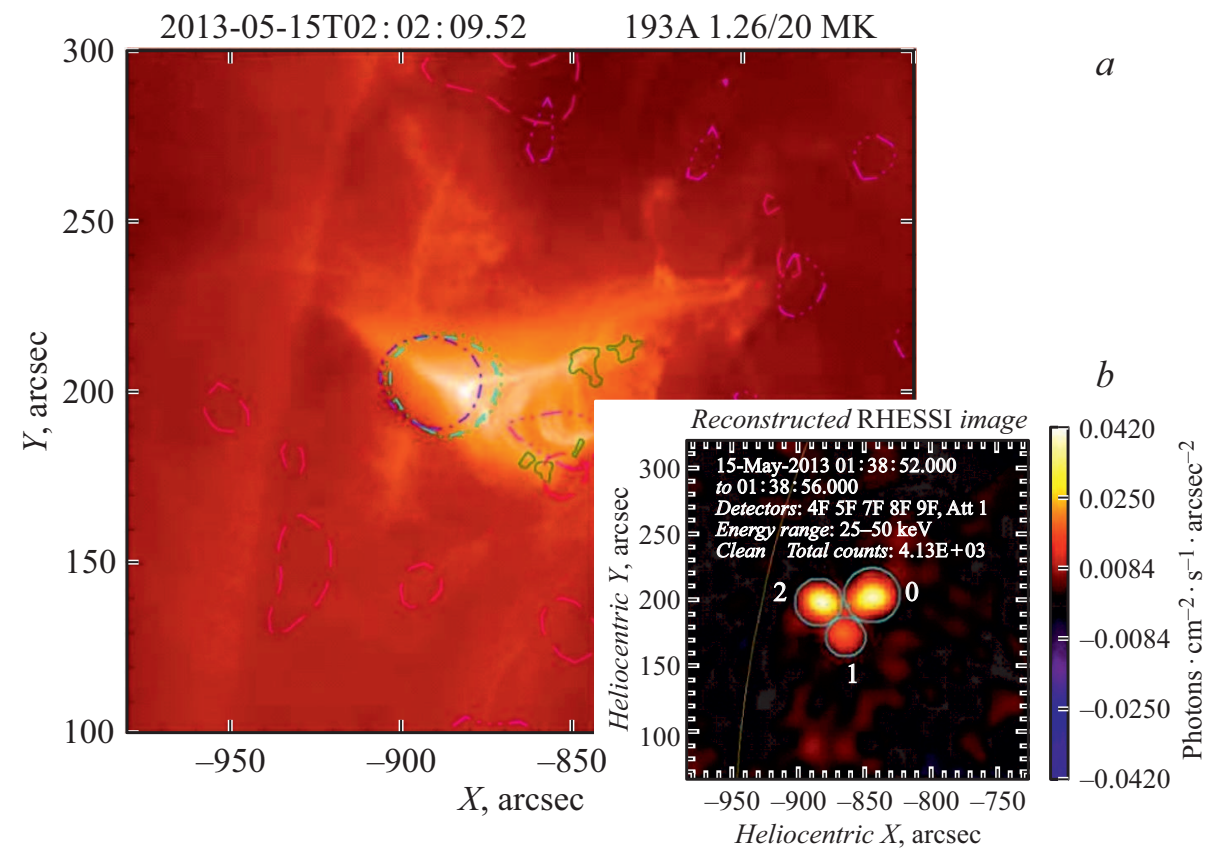

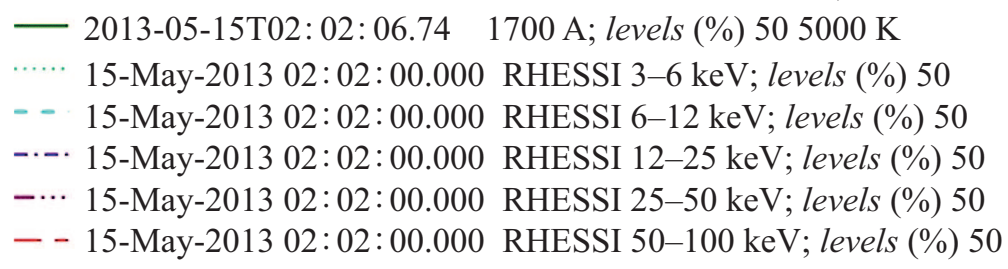

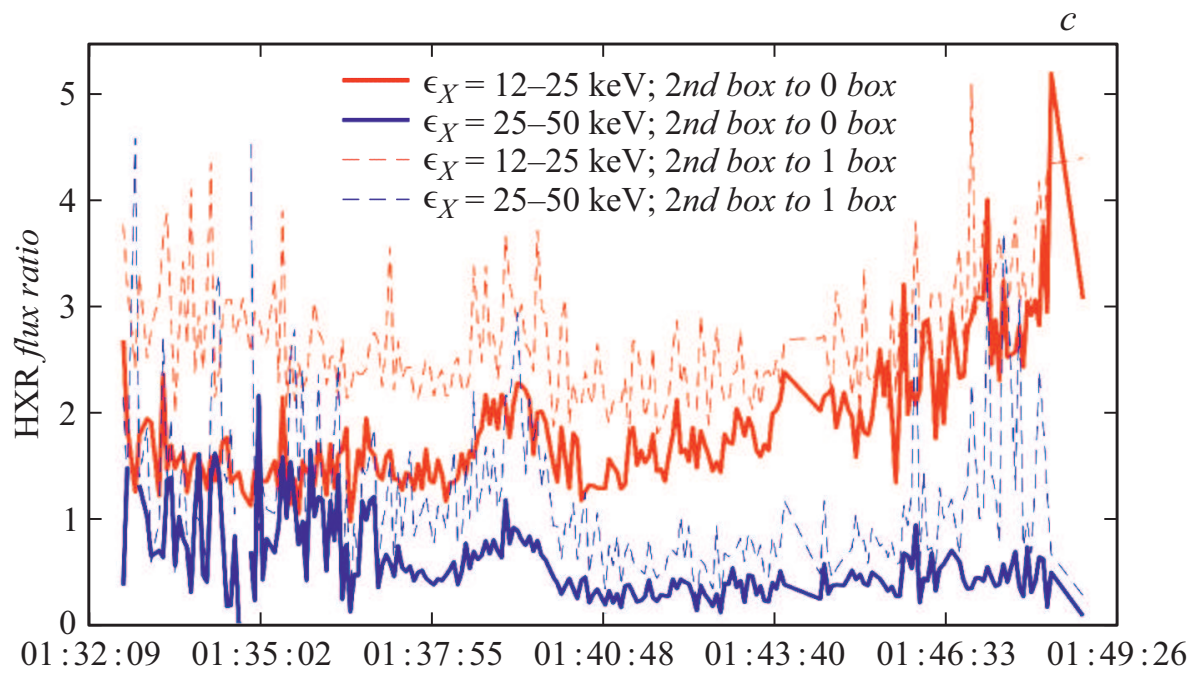

Рис. 1. $a-$ изображение вспышки AIA/SDO 193 А. Подписи к контурным линиям даны в легенде. $b-$ анализируемые боксы. $c-$ соотношение потоков ЖР излучения в боксе 2 к ЖР излучению в боксе 0 и 1 в двух диапазонах энергий.

ле [15,16]. Метод расчета индукционного электрического поля из условия квазинейтральности по току на основе решения кинетического уравнения Фоккера-Планка подробно рассмотрен в нашей более ранней статье [11]. Расчет индукционного электрического поля показан для одной половины магнитной петли на рис. 2. Отметим, что в верхней части петли $s=(0-0.5) \cdot 10^{9} \mathrm{~cm}$ возникают области повышенного электрического поля и аномального сопротивления (модели №№ 2, 5, 8, 10, 12 с ионно-звуковой модой), приводящие к дополнительному угловому рассеянию ускоренных электронов. В анизотропной модели № 10 (черная пунктирная кривая) возникают две близко расположенные области с ионно-звуковой турбулентностью. В зеркальной половине петли распределение абсолютной величины электрического поля аналогично. В моделях без ионно-звуковой турбулентности величина электрического поля в протяженной средней части петли больше, чем в моделях №№ 2, 5, $8,10,12$. Так как индукционное электрическое по- 
Параметры рассмотренных моделей и обозначения кривых для рис. 2-8

\begin{tabular}{|c|c|c|c|c|c|c|c|c|}
\hline Кривая модели на рис. 2-8 & № & Питч-угловое распределение & Показатель спектра $\delta$ & $m$ & $W^{i s}$ & $\delta B / B$ (looptop) & $n_{0}^{\mathrm{LT}}, \mathrm{cm}^{-3}$ & $p$ \\
\hline$\square$ & 1 & $S(\alpha)=1$ & 3 & 2 & 0 & 0 & $10^{10}$ & $\sim 0$ \\
\hline$\square$ & 2 & $S(\alpha)=1$ & 3 & 2 & $10^{-4}$ & 0 & $10^{10}$ & $\sim 0$ \\
\hline$\square$ & 3 & $S(\alpha)=1$ & 3 & 2 & 0 & $5 \cdot 10^{-2}$ & $10^{10}$ & $\sim 0$ \\
\hline 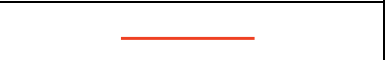 & 4 & $S(\alpha)=1$ & 7 & 2 & 0 & 0 & $10^{10}$ & 0.02 \\
\hline$\longrightarrow$ & 5 & $S(\alpha)=1$ & 7 & 2 & $10^{-4}$ & 0 & $10^{10}$ & -0.02 \\
\hline 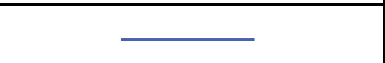 & 6 & $S(\alpha)=1$ & 7 & 2 & 0 & $5 \cdot 10^{-2}$ & $10^{10}$ & -0.01 \\
\hline$\square$ & 7 & $S(\alpha)=1$ & 3 & 2 & 0 & 0 & $8 \cdot 10^{10}$ & $\sim 0$ \\
\hline+ & 8 & $S(\alpha)=1$ & 3 & 7 & $10^{-4}$ & 0 & $10^{10}$ & $\sim 0$ \\
\hline f.............. & 9 & $S(\alpha)=\cos ^{12}(\alpha)$ & 7 & 2 & 0 & 0 & $10^{10}$ & -0.07 \\
\hline f.............. & 10 & $S(\alpha)=\cos ^{12}(\alpha)$ & 7 & 2 & $10^{-4}$ & 0 & $10^{10}$ & -0.02 \\
\hline 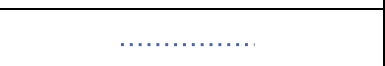 & 11 & $S(\alpha)=\cos ^{12}(\alpha)$ & 7 & 2 & 0 & $5 \cdot 10^{-2}$ & $10^{10}$ & -0.06 \\
\hline 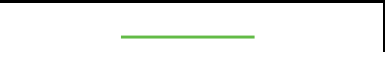 & 12 & $S(\alpha)=1$ & 3 & 2 & $10^{-3}$ & 0 & $10^{10}$ & $\sim 0$ \\
\hline
\end{tabular}

Пр имечан ие. Обозначения слева направо: порядковый номер модели, тип питч-углового распределения и показатель $\delta$ степенного по энергии распределения ускоренных электронов, соотношение максимального значения индукции магнитного поля в основаниях к минимальному в вершине (mirror ratio) $m$, относительный уровень ионно-звуковой турбулентности $W^{i s}=W^{s} / n k_{\mathrm{B}} T_{e}$, уровень магнитных флуктуаций $\delta B / B$ в вершине магнитной петли, концентрация плазмы в вершине $n_{0}^{\mathrm{LT}}$, интегральная по всей петле линейная поляризация $p$ для моделей на рис. 7 . Красным цветом обозначены модели без учета турбулентности. Черным цветом - модели с ионно-звуковой турбулентностью с плотностью энергии в области генерации $W^{s} / n k_{\mathrm{B}} T_{e}=10^{-4}$, синим цветом - модели с учетом магнитных флуктуаций, зеленым - модель с повышенным уровнем $W^{s} / n k_{\mathrm{B}} T_{e}=10^{-3}$. Сплошные кривые отвечают изотропным моделям, пунктирные - анизотропным. Квадратные маркеры относятся к моделям с группой параметров $\delta=3, m=2$; без маркеров $-\delta=7, m=2$; большой квадратный маркер - модель с высоким значением $n_{0}^{\mathrm{LT}}$; маркер в виде креста $-\delta=3, m=7$. Поток энергии электронов в момент максимума инжекции во всех моделях составляет $10^{11} \mathrm{erg} /\left(\mathrm{cm}^{2} \cdot \mathrm{s}\right)$. Инжекция ускоренных электронов в верхней части петли задана в виде $S(t)=\exp \left[-\left(t-t_{1}\right)^{2} / t_{0}^{2}\right]$, где $t_{1}=2.6 \mathrm{~s}, t_{0}=1.4 \mathrm{~s}$.

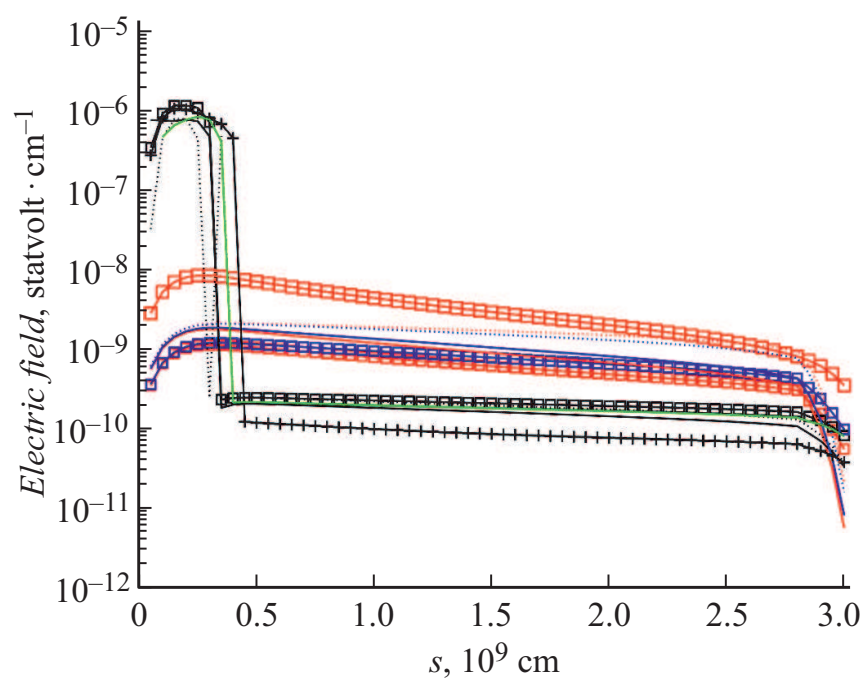

Рис. 2. Распределение индукционного электрического поля вдоль одной половины магнитной петли для моделей, приведенных в таблице, в момент максимума инжекции $t=2.6 \mathrm{~s}$.

ле также оказывает влияние на питч-угловое распределение быстрых электронов, этот факт оказывается существенным при интерпретации данных моделирования.
Для рассмотренных моделей (см. таблицу) результаты расчета функции распределения вдоль петли для времени максимума потока и для энергии электронов $30 \mathrm{keV}$ представлены на рис. 3. Существенным на рисунке является не только плотность ускоренных электронов в корональной части петли, но также и в

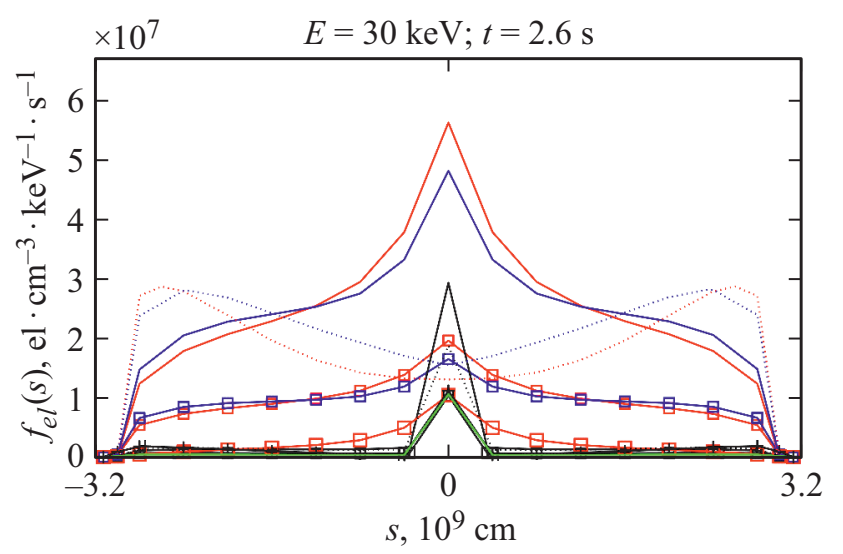

Рис. 3. Функция распределения ускоренных электронов вдоль вспышечной петли для энергии электронов $E=30 \mathrm{keV}$, проинтегрированная по питч-углам. Рисунок соответствует времени максимума инжекции. Набор кривых соответствует моделям из таблицы. 
основаниях - в крайних точках графика, так как в этих точках плотность плазмы выше на 3-5 порядков и достигает значений $10^{15} \mathrm{~cm}^{-3}$, что определяет поток ЖР излучения из оснований.

Первоначально сравним изотропные модели (№ 1-8 и 12). Кривые для моделей № 4, 6 (красная и синяя кривые (в online версии)) и № 1, 3 (красные и синие квадраты (в online версии)) практически не различаются между собой в фазе максимума, что свидетельствует о незначительном влиянии рассеяния на магнитных флуктуациях с заданным уровнем в этой фазе вспышки. В изотропных моделях №№ 2, 5, 8, 12 (черные и зеленая кривые (в online версии)) распределения электронов обнаруживают пики, локализованные в вершине, но при этом возникают „провалы“ в средней части петли. Этот результат можно объяснить захватом ускоренных электронов в области с ионно-звуковой турбулентностью, а также их частичным перераспределением из средней части петли в вершину. Высокая концентрация плазмы в вершине (модель № 7), наоборот, приводит к более широкому распределению электронов в корональной части петли. В анизотропных моделях №№ 9, 11 при отсутствии ионно-звуковой моды пик в распределении электронов в вершине петли не прослеживается (красные и синие пунктирные кривые (в online версии)), поскольку квазипродольно распределенные электроны $\left(f \sim \cos ^{12} \alpha\right)$ в момент инжекции преимущественно уходят в конус потерь, не успевая эффективно рассеяться на кулоновских соударениях и эффективно отразиться от магнитных зеркал с отношением $m=2$. В анизотропной модели № 10 с ионно-звуковой турбулентностью (черная пунктирная кривая) распределение ускоренных электронов имеет ярко выраженный максимум в области вершины, в отличие от моделей № 9, 11, подтверждая эффективность процесса рассеяния быстрых электронов на ионно-звуковых плазмонах.

Зависимость функции распределения электронов от питч-угла в корональной части петли представлена на рис. 4. В изотропных моделях № 2, 5, 8, 12 (черные и зеленая кривые (в online версии)) ионно-звуковая турбулентность приводит к формированию более узкого квазипоперечного распределения с максимумом в 90 градусов в сравнении с моделями №№ 1, 3, 4, 6 (красная и синяя кривые (в online версии)) без учета этой моды. Повышенная концентрация в корональной части петли (модель № 7) или присутствие магнитных флуктуаций (модели № 3, 6) в изотропных моделях размывают квазипоперечное распределение. В анизотропной модели (пунктирные кривые) дополнительное рассеяние на ионно-звуковой турбулентности формирует плато в области питч-углов 50-120 градусов (модель № 10) в сравнении с анизотропной моделью без турбулентности № 9 и моделью с магнитными флуктуациями № 11 .

Зависимость функции распределения электронов от питч-угла в „правом“ основании петли дана на рис. 5. Питч-углы < 90 градусов отвечают электронам, которые распространяются в направлении атмосферы Солнца.

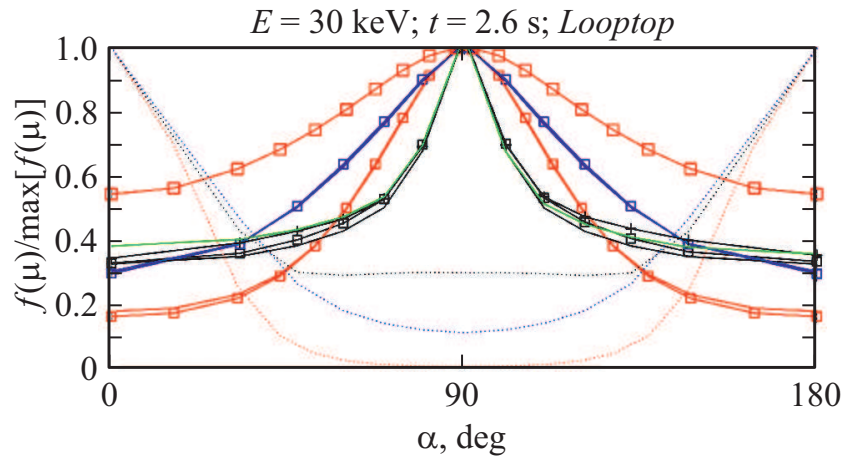

Рис. 4. Питч-угловая $(\mu=\cos (\alpha))$ зависимость дифференциальной по энергии функции распределения электронов, проинтегрированной по области петли в вершине $s=(-1.5-1.5) \cdot 10^{9} \mathrm{~cm}$. Рисунок соответствует времени максимума инжекции. Подписи к кривым даны в таблице.

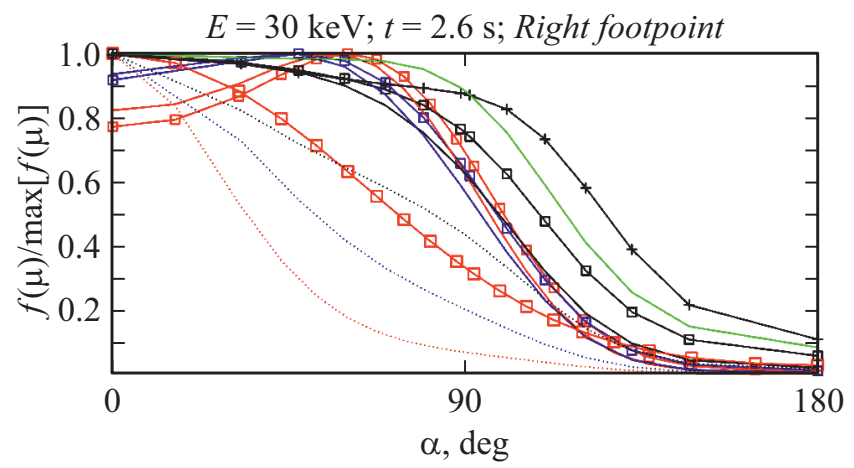

Рис. 5. Питч-угловая $(\mu=\cos (\alpha))$ зависимость дифференциальной по энергии функции распределения электронов, проинтегрированной по области петли в основании $s=(2-3) \cdot 10^{9} \mathrm{~cm}$. Рисунок соответствует времени максимума инжекции. Подписи к кривым даны в таблице.

Наиболее квазипродольные распределения соответствуют анизотропным моделям № 9-11 (пунктирные кривые) и изотропной модели с высокой концентрацией плазмы в вершине № 7. Наиболее квазипоперечные распределения в основаниях петли обнаруживаются у всех изотропных моделей, особенно в модели с высоким градиентом магнитного поля № 8 .

Интенсивность рентгеновского излучения рассчитывается согласно формулам релятивистского тормозного излучения [6] электрона при торможении в электрическом поле протона. Свертка функции распределения ускоренных электронов с сечением тормозного рассеяния для различных моделей определяет поток ЖР излучения. На рис. 6 приведены распределения ЖР излучения для угла наблюдения 90 градусов (лимбовые события) в области энергий 29-58 keV. Заметим, что кривые ЖР излучения по характеру подобны кривым распределения функции распределения ускоренных электронов, приведенным на рис. 3. В изотропных моделях №№ 2, 5, 8, 12 (черная и зеленая кривые (в online 


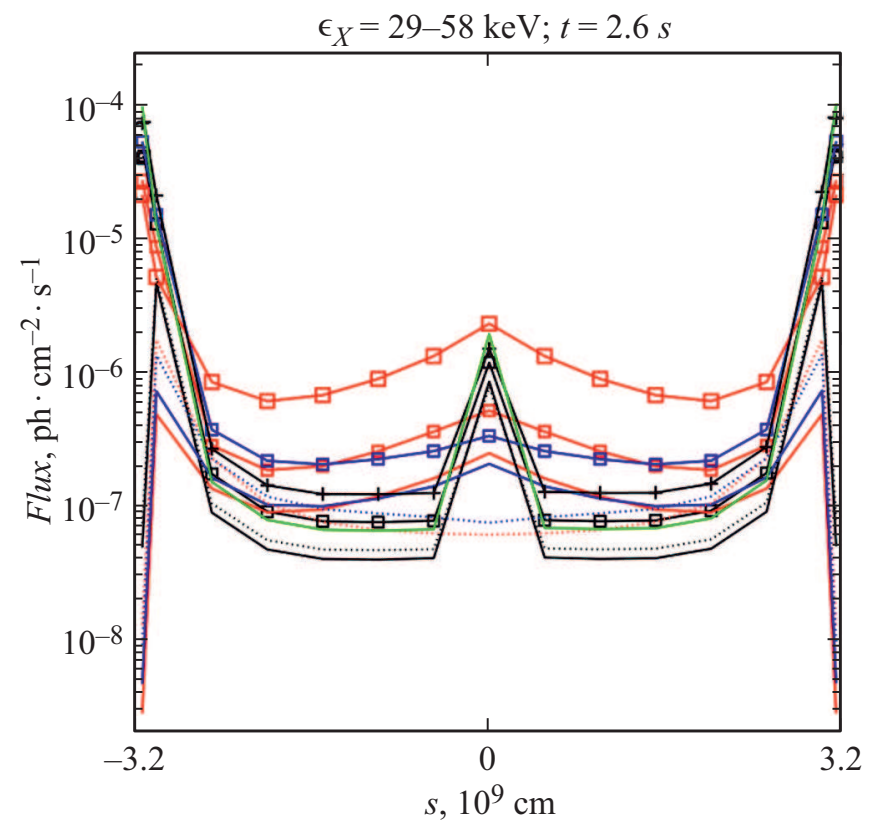

Рис. 6. Проинтегрированное по сечению магнитной петли распределение ЖР излучения вдоль петли. Угол наблюдения 90 градусов. Рисунок соответствует времени максимума инжекции. Подписи к кривым даны в таблице.

версии)) и в анизотропной модели № 10 (черная пунктирная кривая) с ионно-звуковой турбулентностью обнаруживаются компактные области ЖР излучения в вершине петли. Повышенная концентрация плазмы в петле также приводит к яркой более протяженной области излучения в вершине и к менее ярким основаниям (модель № 7).

На рис. 7, $a$ представлены кривые соотношения ЖР излучения в вершине к сумме ЖР излучения в вершине и двух основаниях, т.е. фактически вклад излучения из вершины в общее излучение вспышечной петли. Рассмотрим модели с ионно- звуковой турбулентностью (черные кривые). Ввиду различных распределений ускоренных электронов по питч-углу и энергии в рассмотренных моделях (см. таблицу), динамика индукционного электрического поля различается. В результате, в небольших пределах отличается время и область пространства, при которых происходит генерация ионнозвуковой турбулентности. На рис. 7, $b$ приведен пример изменения индукционного электрического поля во времени и пространстве для модели № 2. Генерация турбулентности в изотропной модели № 2 началась примерно в момент времени $t \sim 1 \mathrm{~s}$. В случае анизотропной модели № 10 и изотропной модели № 5 с мягким спектром $\delta=7-$ немного раныше, в момент $t \sim 0.8 \mathrm{~s}$, а в случае модели № 8 с параметром $m=7$ - немного позже, в момент $t \sim 1.2 \mathrm{~s}$. В это время кривые относительного вклада ЖР излучения из вершины испытывают резкое изменение - падение в случае изотропных моделей №№ 2, 5, 8, 12, что свидетельствует об эффективном рассеянии ускоренных электронов в области генерации ионного звука и росту в случае анизотропной модели № 10. Последнее связано также с эффективным рассеянием электронов, но поскольку в этой модели распределение электронов по питч-углам изначально преимущественно квазипродольное, дополнительное рассеяние приводит к эффективному захвату электронов в первые $\sim 0.2 \mathrm{~s}$ от момента начала генерации ионно-звуковой турбулентности. В дальнейшем доля ЖР излучения из вершины после стремительного роста также стремительно уменьшается в момент времени $t \sim 1.1 \mathrm{~s}$. Это вызвано более сложной картиной захвата и рассеяния электронов - после первоначального захвата электронов и формирования квазипоперечного распределения электроны продолжают рассеиваться на турбулентности, что, в конечном счете, приводит к их „вымыванию“ из области вершины. Кроме того, в данной модели (№ 10) возникают две близкорасположенные области с ионно-звуковой турбулентностью (рис. 2), что дополнительно усложняет картину распространения электронов. В другой половине петли происходит симметричное раздвоение

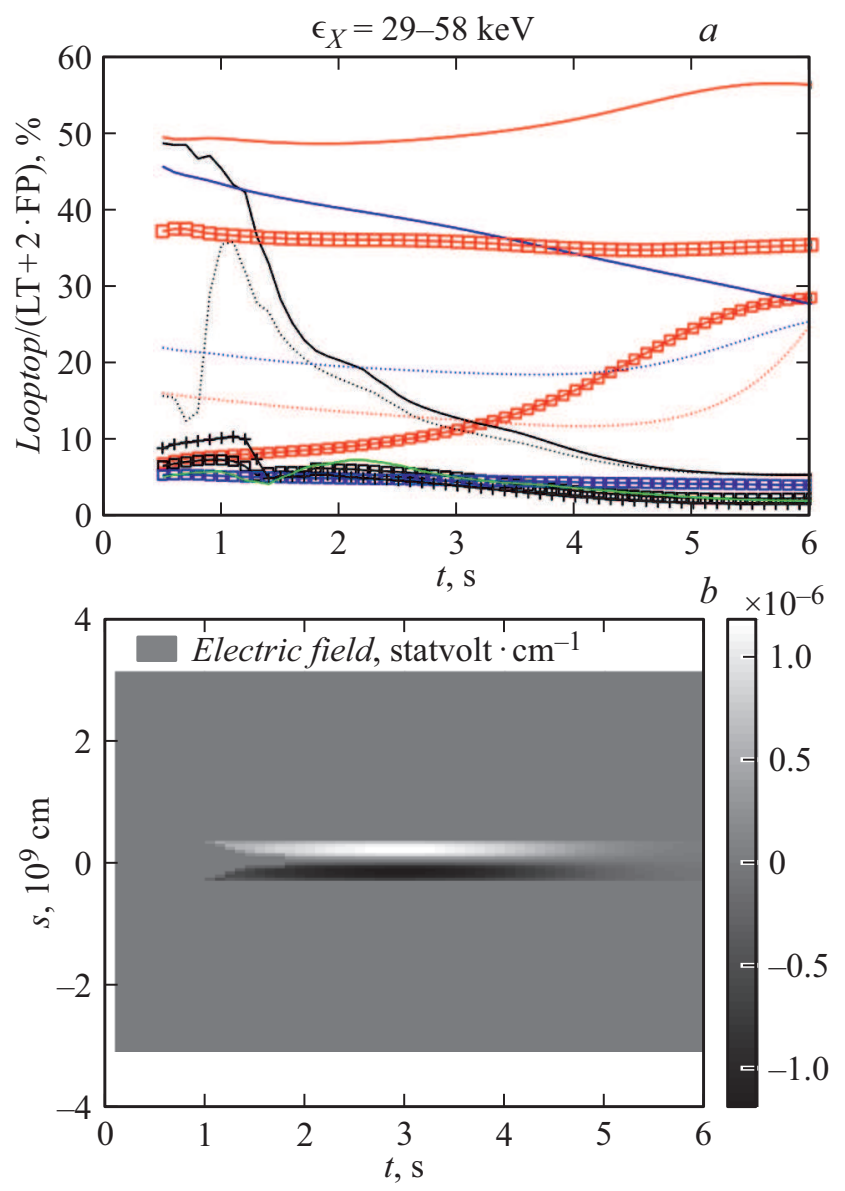

Рис. 7. $a-$ зависимость от времени соотношения ЖР излучения в вершине к сумме ЖР излучения в вершине и двух основаниях. Потоки в вершине получены интегрированием по корональной части петли $(-1.5-1.5) \cdot 10^{9} \mathrm{~cm} . b-$ распределение индукционного электрического поля во времени и пространстве для модели № 2. 
области с ионно-звуковой турбулентностью. Обратим внимание, что наиболее высокую яркость вершины (доля более $30 \%$ ) обеспечивают изотропные модели с мягким энергетическим спектром ускоренных электронов $\delta=7$ (модели №№ 4-6), анизотропная модель с ионнозвуковой турбулентностью (модель № 10), а также модель с высокой концентрацией плазмы в вершине (модель № 7). Таким образом, области с ионно-звуковой турбулентностью и в меньшей степени магнитные флуктуации в изотропных моделях, наоборот, приводят к уменьшению яркости вершины (см. черные кривые). Компактные области ЖР излучения, обнаруживаемые на рис. 6 в моделях с ионно-звуковой турбулентностью, имеют малую меру эмиссии и с наблюдательной точки зрения, при анализе вспышки на диске, вряд ли могут быть обнаружены вследствие малого динамического диапазона существующих на сегодняшний день спектрометров с пространственным разрешением. Например, сравним долю ЖР излучения из вершины на рис. 7 в моделях № 1 и 2 (красные и черные квадраты (в online версии)). В момент времени $t=2.6 \mathrm{~s}$, соответствующий моменту времени для распределений ЖР излучения на рис. 6, доля ЖР излучения в модели без турбулентности составляет $\sim 10 \%$ (рис. 7 ), а в модели с ионно-звуковой турбулентностью - около 5\%. До $t \sim 1 \mathrm{~s}$ яркость вершины в этих моделях совпадает, так как ионно-звуковая турбулентность начала генерироваться после $t \sim 1 \mathrm{~s}$. Еще более наглядным примером являются изотропные модели № 4 и 5 . В момент времени $t \sim 2.6 \mathrm{~s}$ в модели с ионно-звуковой турбулентностью доля ЖР излучения из вершины составляет $\sim 15 \%$ (черная сплошная кривая) против $\sim 50 \%$ (красная сплошная кривая (в online версии)) в модели без турбулентности. На спектрометрах с пространственным разрешением более 10-20 угловых секунд даже в случае, если бы динамического диапазона было достаточно для наблюдения источника с яркостью на уровне $5 \%$ от интегрального излучения, компактная область, например, такая, как в модели № 2 (рис. 6) будет слабо отличимой от более диффузного коронального источника в модели № 1. Таким образом, по яркости и локализации излучения из вершины в ЖР диапазоне $25-50 \mathrm{keV}$ различить изотропные модели с ионно-звуковой турбулентностью и без турбулентности не представляется возможным. Усиленный захват электронов вследствие высокого градиента магнитного поля (модель № 8) не приводит к существенному росту яркости вершины, как и более высокий уровень ионно-звуковой турбулентности $W^{s} / n k_{\mathrm{B}} T_{e}=10^{-3}$ (модель № 12, зеленая кривая (в online версии)). В изотропных моделях с жестким спектром электронов $\delta=3$ (№ 1-3) вне зависимости от присутствия магнитных флуктуаций или ионно-звуковой турбулентности доля ЖР излучения из вершины составляет менее $10 \%$. Примечательно, что яркость ЖР излучения из вершины петли оказывается больше в анизотропных моделях с мягким энергетическим спектром ускоренных электронов $\delta=7$ и/или с турбулентностью (модели № 9-11,

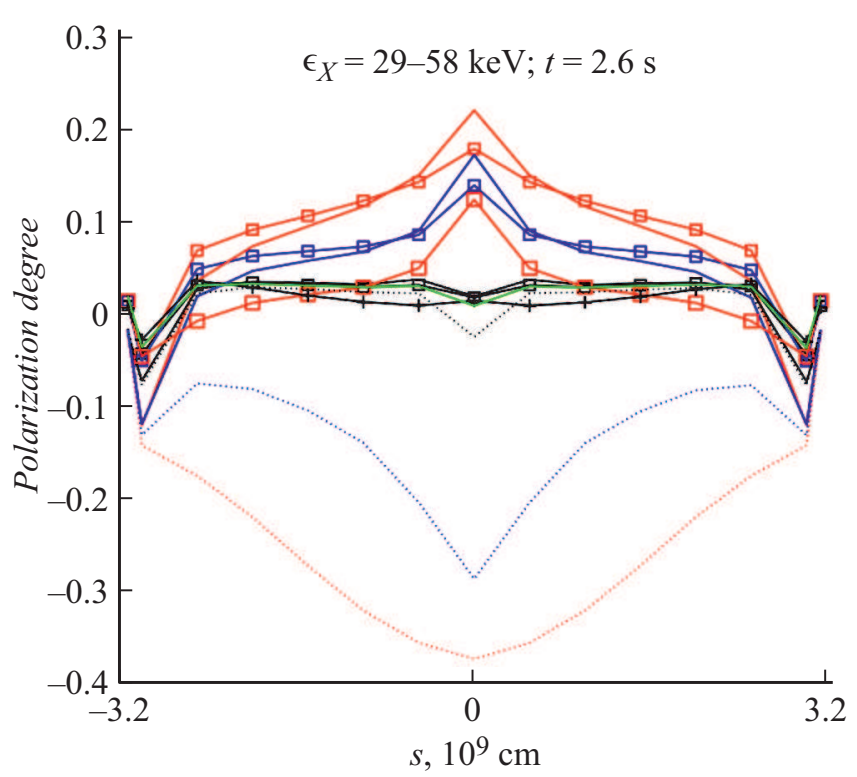

Рис. 8. Распределение линейной степени поляризации ЖР излучения вдоль петли. Угол наблюдения 90 градусов. Рисунок соответствует времени максимума инжекции. Подписи к кривым даны в таблице.

пунктирная кривая). Захвату электронов в данных моделях способствует как кулоновское рассеяние, так и рассеяние индукционным электрическим полем.

Распределение линейной степени поляризации ЖР излучения вдоль петли в диапазоне энергий $29-58 \mathrm{keV}$ дано на рис. 8. Как видно, в моделях с ионно- звуковой турбулентностью степень поляризации в вершине близка к нулю (черные и зеленая кривые (в online версии)). В моделях без турбулентности пиковые значения достигают величин $\sim+22 \%$ и $\sim-36 \%$ в изотропной (модель № 4) и анизотропной моделях (№ 9) соответственно. В обоих случаях спектр электронов мягкий с показателем спектра $\delta=7$. Так как на данный момент времени отсутствуют приборы, способные измерять степень поляризации в ЖР диапазоне с высоким пространственным разрешением, необходимо привести интегральные значения степени поляризации, которые даны в правой колонке таблицы. В этом случае в модели № 4 степень поляризации уменьшается до значения $+2 \%$, а в модели № 9 - до значения $-7 \%$.

\section{Заключение}

Результаты численных расчетов позволяют заключить, что присутствие дополнительных к кулоновскому механизмов рассеяния ускоренных электронов в ограниченной области корональной части магнитной петли не всегда приводит к возникновению яркого источника ЖР излучения в вершине. Исключением является случай c анизотропной инжекцией на начальном этапе, когда эффективность накопления электронов за счет захвата 
квазипродольных электронов в магнитной петле в результате дополнительного рассеяния превышает эффективность рассеяния квазипоперечных, ранее захваченных, электронов. Не столь важным оказывается и высокий градиент магнитного поля. Кулоновский механизм рассеяния в изотропных моделях в сочетании с мягким степенным спектром электронов с показателем $\delta \gtrsim 6$ и/или относительно высокой концентрацией плазмы в вершине петли с $n_{0}^{\mathrm{LT}} \gtrsim 10^{10} \mathrm{~cm}^{-3}$ оказывается весьма эффективным при генерации корональных источников ЖР излучения. Особенно ярко это проявляется на фазе спада, когда уже нельзя пренебрегать эффектом испарения плазмы из хромосферы [17]. Если же энергетический спектр электронов жесткий с $\delta=3$, то вне зависимости от присутствия турбулентности доля ЖР излучения из вершины составляет менее $10 \%$ от интегральной. Так же отметим, что яркость ЖР излучения из вершины петли в анизотропной модели с мягким энергетическим спектром ускоренных электронов $\delta=7$ (модель № 9, пунктирная кривая) оказывается выше, чем в изотропной модели № 1 с жестким энергетическим спектром с $\delta=3$, что ранее также было показано в работе с учетом только кулоновского рассеяния [18], т. е. в случае мягкого спектра, рассеяние электронов при кулоновских соударениях и на индукционном электрическом поле оказывается очень эффективным. Отметим, что во всех моделях с локализованной ионно-звуковой турбулентностью возникает характерный компактный источник ЖР излучения в вершине. При сравнении моделей с ионно- звуковой турбулентностью и без турбулентности можно заключить, что перераспределение ускоренных электронов в петле, в результате рассеяния на турбулентности и под влиянием повышенного индукционного электрического поля в областях с аномальным сопротивлением, приводит к уменьшению как пространственных размеров коронального источника, так и его яркости. Вывод справедлив для потоков, полученных интегрированием по корональной части петли $(-1.5-1.5) \cdot 10^{9} \mathrm{~cm}$. Превышение яркости вершины в моделях с турбулентностью в сравнении с моделями без турбулентности происходит в очень узкой области вблизи координаты $s \sim 0 \mathrm{~cm}$. Пространственное разрешение существующих спектрометров не достаточно для уверенной диагностики и разделения изотропных моделей с турбулентностью и без турбулентности по данному признаку. Экстремальные значения линейной степени поляризации ЖР излучения вдоль петли на энергиях $29-58 \mathrm{keV}$ в присутствии турбулентности уменьшаются на $\sim 5-35 \%$. Интегральные значения поляризации от всей магнитной петли, даже без дополнительного рассеяния электронов, не превышают по модулю 10\%. Наблюдения линейной поляризации ЖР излучения более $10 \%$ возможны только для корональных источников, что, как правило, означает регистрацию излучения от залимбовых событий.

\section{Конфликт интересов}

Авторы заявляют, что у них нет конфликта интересов.

\section{Список литературы}

[1] P. Saint-Hilaire, S. Krucker, R.P. Lin. Sol. Phys., 250 (1), 53 (2011). DOI: 10.1007/s11207-008-9193-9

[2] S. Masuda, T. Kosugi, H. Hara, S. Tsuneta, Y. Ogawara. Nature, 371, 495 (1994). DOI: 10.1038/371495a0

[3] S. Masuda, T. Kosugi, H. Hara, T. Sakao, K. Shibata, S. Tsuneta. Publ. Astron. Soc. Jpn., 47 (5), 677 (1995).

[4] M. Tomczak, T. Ciborski. Astron. Astrophys., 461, 315 (2007). DOI: 10.1051/0004-6361:20066115

[5] Y.W. Jiang, S. Liu, W. Liu, V. Petrosian. Astrophys. J., 638 (2), 1140 (2005). DOI: $10.1086 / 498863$

[6] T. Bai, R. Ramaty. Astrophys. J., 219, 705 (1978). DOI: $10.1086 / 155830$

[7] E.P. Kontar, N.H. Bian, A.G. Emslie, N. Vilmer. Astrophys. J., 780, 176 (2014). DOI: 10.1088/0004-637X/780/2/176

[8] V.F. Melnikov, Y.E. Charikov, I.V. Kudryavtsev. Geomagn. Aeron., 55 (7), 983 (2015). DOI: $10.1134 / \mathrm{S} 0016793215070130$

[9] T.D. Arber, V.F. Melnikov. Astrophys. J., 690 (1), 238 (2009). DOI: $10.1088 / 0004-637 \mathrm{X} / 690 / 1 / 238$

[10] J.C. Brown, D.B. Melrose, D.S. Spicer. Astrophys. J., 228, 592 (1979). DOI: $10.1086 / 156883$

[11] Y.E. Charikov, A.N. Shabalin. Geomagn. Aeron., $56(8), 1068$ (2016). DOI: 10.1134/S0016793216080041

[12] В.Н. Цытович. Нелинейные эффекты в плазме (Наука, M., 1967)

[13] В.Н. Цытович. Теория турбулентной плазмы (Атомиздат, М., 1971)

[14] И.В. Кудрявцев, Ю.Е. Чариков. Астроном. журн., 68 (4-6), 825 (1991).

[15] H.L. Rowland, L. Vlahos. Astron. Astrophys., 142 (2), 219 (1985).

[16] G.H.J. van den Oord. Astron. Astrophys., $234(1-2), 496$ (1990).

[17] J.C. Allred, S.L. Hawley, W.P. Abbett, M. Carlsson. Astrophys. J., 630 (1), 573 (2005). DOI: 10.1086/431751

[18] V.F. Melnikov, Y.E. Charikov, I.V. Kudryavtsev. Geomagn. Aeron., 53 (7), 863 (2013). DOI: $10.1134 / \mathrm{S} 0016793213070153$ 